\title{
Myotonic dystrophy complicated by peripheral vascular disease
}

\author{
A. S. David \\ M.B., Ch.B.
}

R. O. QUIN

M.D., F.R.C.S.

Gartnaval General Hospital, 1053 Great Western Road, Glasgow G12 OYN

\begin{abstract}
Summary
A case of myotonic dystrophy complicated by peripheral vascular disease is described. No obvious explanation was found to account for this. A review of the literature revealed a few similar cases. The possibility that disordered peripheral circulation may be a rare feature of myotonic dystrophy is proposed.

\section{Introduction}

Myotonic dystrophy was first described in this country by Batten and Gibb in 1909. In 1911, Griffith drew attention to the associated cardiological complications. Since then, many abnormalities outside the neuromotor system such as multiple endocrinopathies have been described in this condition. The following case was complicated by peripheral vascular disease.
\end{abstract}

\section{Case report}

A 40-year-old man was admitted to a surgical unit with a 3-week history of increasing pain in the right foot. In the past year he had experienced pain in both calves on walking half a mile, and in addition, had been falling frequently, his legs ' $s$ 'ving way'. He had had bilateral cataract extractions on: year previously. There was no history of diabetes melitus or medication. He was an unmarried, unemploy id labourer, a non-smoker but a drinker of at least tu o pints of beer thrice a week. There was no relevant $f_{f}$.mily history available.

On examination, he had the characteristic featu: es of myotonic dystrophy (Fig. 1), including sterncmastoid, facial and distal limb weakness and wasting, plus percussion myotonia. There was also hypogonadism and gynaecomastia. The pulse was irregular, the blood pressure $125 / 90 \mathrm{mmHg}$. Peripheral pulses were absent below the femorals bilaterally. The right foot was the cooler and there was ischaemic gangrene affecting the great toe (Fig. 2).

\section{Investigations}

Full blood count, urea and electrolytes, glucose

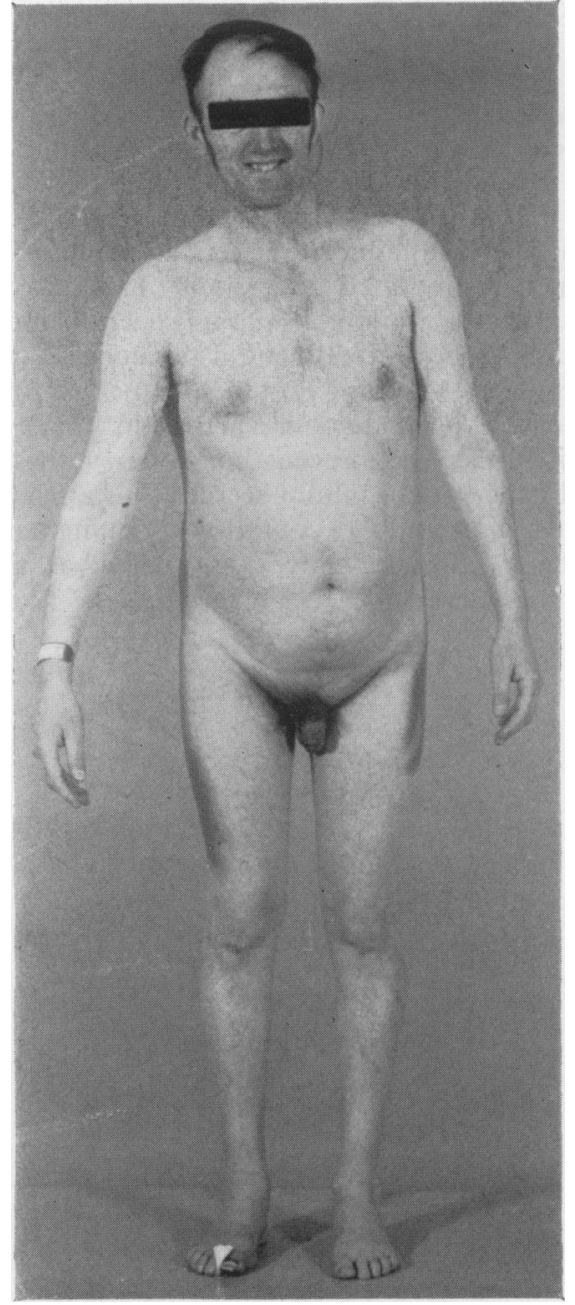

Fig. 1. Showing some of the characteristic features of myotonic dystrophy including frontal baldness, facial, sternomastoid and distal limb wasting. Also note gynaecomastia and hypogonadism. 
profile, fasting lipids, creatine kinase and chest $\mathrm{X}$-ray were normal. Erythrocyte sedimentation rate (ESR) $17 \mathrm{~mm} / \mathrm{hr}$. Liver function tests revealed raised serum aspartate and alanine aminotransferase, alkaline phosphatase and gamma glutamyl transferase concentrations. Serum IgG $6.9 \mathrm{~g} / \mathrm{l}$ (normal 7-19). Serum luteinizing hormone and follicle stimulating hormone were elevated and testosterone levels were subnormal.

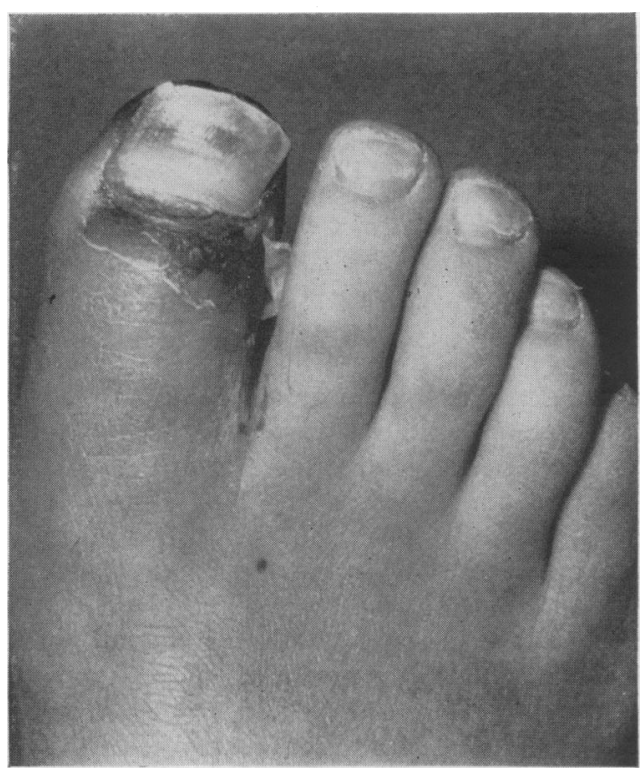

Fig. 2. The right foot affected by ischaemic gangrene of the great toe with a line of demarcation through the proximal phalanx.

Serological tests for syphilis and connective tissue disease were negative. Chromosome analysis gave a male karyotype. An isotope brain scan was normal, but the verbal IQ was just below normal.

The electrocardiogram showed atrial flutter with variable block. An arteriogram revealed patchy atheromatous narrowing of the aortic bifiurcation in both iliac and femoral arteries. The right popliteal artery was stenosed at the origin of the anterior tibial. All three major vessels in the calf showed atheromatous change.

The hormonal abnormalities, those of 'endorgan failure', the low IgG, and cardiac conduction defects of the kind noted are all consistent with the syndrome of myotonic dystrophy, as is intellectual impairment. No common predisposing factors to atheroma were present in that the patient had no diabetic tendency nor abnormal lipids and he was a non-smoker. The ESR was normal, excluding arteritis. The hepatic dysfunction improved and was presumed to be due to alcohol abuse.

The patient was discharged, leaving his toe to auto-amputate.

\section{Discussion}

Sporadic cases of disordered peripheral circulation in myotonic dystrophy have been published. Waring, Ravin and Walker (1940) in a review of 13 cases found 10 complaining of cold hands and feet with cyanosis of varying degrees; this is however a common finding in peripheral nerve and muscular disease. Leinwand (1948) reported a 48-year-old man, a smoker, who complained of right calf claudication and was without pulses below the right femoral. Another case was presented by Mahoudeau et al. (1956) of a 37-year-old male who had no right radial or dorsalis pedis pulse. Variable loss of other pulses occurred and thrombo-embolism secondary to arrhythmias was one hypothesis to explain this. More recently, 'small vessel disease' in the shape of Raynaud's phenomenon has been noted (Agrawal et al., 1975).

It is probably justifiable to propose tentatively, that abnormalities in the peripheral circulation are a rare complication of myotonic dystrophy.

\section{Acknowledgment}

The authors wish to thank Mrs F. Bickerton for her secretarial work.

\section{References}

Agrawal, A.K., Ambastha, S.P., Prasad, R. \& Sinha, K.K. (1975) Myotonic dystrophy with Raynaud's phenomena. Journal of the Association of Physicians of India, 23, 377.

Batten, F.E. \& GibB, H.P. (1909) Myotonia atrophica. Brain, 32, 187.

GRIFFITH, T.W. (1911) On myotonia. Quarterly Journal of Medicine, 5, 229.

LEINWAND, I. (1948) Dystrophia myotonia complicated by thromboangiitis obliterans and pseudohypertrophic muscular dystrophy. New York State Journal of Medicine, 48, 1503.

Mahoudeau, D., Delamare, J., Daum, S. \& Dubrisay, J. (1956) Les manifestations cardiovasculaire de la maladie de Steinert. Bulletins et Memoires de la Société Médicale de Hopitaux de Paris, 72, 383.

WARING, J.J., Ravin, A. \& Walker, C.E.(1940) Dystrophia myotonica; a review of 13 cases. Archives of Internal Medicine, 65, 763. 